Draft version December 8, 2017

Preprint typeset using $\mathrm{L}_{\mathrm{A}}^{\mathrm{T}} \mathrm{X}$ style emulateapj v. 12/16/11

\title{
ALMA REVEALS TRANSITION OF POLARIZATION PATTERN WITH WAVELENGTH IN HL TAU'S DISK
}

\author{
Ian W. Stephens ${ }^{1}$, Haifeng Yang ${ }^{2}$, Zhi-Yun Li ${ }^{2}$, Leslie W. Looney ${ }^{3}$, Akimasa Kataoka ${ }^{4,5}$, Woojin Kwon ${ }^{6,7}$, \\ Manuel Fernández-López ${ }^{8}$, Charles L. H. Hull ${ }^{5,9,10}$, Meredith Hughes ${ }^{11}$, Dominique SeGura-Cox ${ }^{3,12}$, Lee \\ Mundy ${ }^{13}$, Richard CRutcher ${ }^{3}$, Ramprasad RAO ${ }^{14}$ \\ Draft version December 8, 2017
}

\begin{abstract}
The mechanism for producing polarized emission from protostellar disks at (sub)millimeter wavelengths is currently uncertain. Classically, polarization is expected from non-spherical grains aligned with the magnetic field. Recently, two alternatives have been suggested. One polarization mechanism is caused by self-scattering from dust grains of sizes comparable with the wavelength, while the other mechanism is due to grains aligned with their short axes along the direction of radiation anisotropy. The latter has recently been shown as a likely mechanism for causing the dust polarization detected in HL Tau at $3.1 \mathrm{~mm}$. In this paper, we present ALMA polarization observations of HL Tau for two more wavelengths: $870 \mu \mathrm{m}$ and $1.3 \mathrm{~mm}$. The morphology at $870 \mu \mathrm{m}$ matches the expectation for self-scattering, while that at $1.3 \mathrm{~mm}$ shows a mix between self-scattering and grains aligned with the radiation anisotropy. The observations cast doubt on the ability of (sub)millimeter continuum polarization to probe disk magnetic fields for at least HL Tau. By showing two distinct polarization morphologies at $870 \mu \mathrm{m}$ and $3.1 \mathrm{~mm}$ and a transition between the two at $1.3 \mathrm{~mm}$, this paper provides definitive evidence that the dominant (sub)millimeter polarization mechanism transitions with wavelength. In addition, if the polarization at $870 \mu \mathrm{m}$ is due to scattering, the lack of polarization asymmetry along the minor axis of the inclined disk implies that the large grains responsible for the scattering have already settled into a geometrically thin layer, and the presence of asymmetry along the major axis indicates that the HL Tau disk is not completely axisymmetric.
\end{abstract}

Keywords: protoplanetary disks - polarization - stars: formation - stars: protostars - dust

\section{INTRODUCTION}

Spinning dust grains in the interstellar medium are expected to align with their short axes perpendicular to the magnetic field as a result of radiative torques (Lazarian 2007; Andersson et al. 2015). Given this alignment, the polarization of thermal dust emission at millimeter and submillimeter wavelengths is expected to be perpendicular to the magnetic field. Therefore, polarimetric observations at these wavelengths have frequently been used

${ }^{1}$ Harvard-Smithsonian Center for Astrophysics, 60 Garden Street, Cambridge, MA, USA; ian.stephens@cfa.harvard.edu

2 Astronomy Department, University of Virginia, Charlottesville, VA 22904, USA

${ }^{3}$ Department of Astronomy, University of Illinois, 1002 West Green Street, Urbana, IL 61801, USA

${ }^{4}$ National Astronomical Observatory of Japan, Mitaka, Tokyo 181-8588, Japan

5 NAOJ Fellow

${ }^{6}$ Korea Astronomy and Space Science Institute, 776 Daedeokdaero, Yuseong-gu, Daejeon 34055, Korea

${ }^{7}$ Korea University of Science and Technology, 217 Gajang-ro, Yuseong-gu, Daejeon 34113, Korea

${ }^{8}$ Instituto Argentino de Radioastronomía, (CCT-La Plata, CONICET; CICPBA), C.C. No. 5, 1894, Villa Elisa, Argentina

${ }^{9}$ National Astronomical Observatory of Japan, NAOJ Chile Observatory, Alonso de Córdova 3788, Office 61B, 7630422, Vitacura, Santiago, Chile

10 Joint ALMA Observatory, Alonso de Córdova 3107, Vitacura, Santiago, Chile

${ }^{11}$ Van Vleck Observatory, Astronomy Department, Wesleyan University, Middletown, Connecticut 06459, USA

12 Max-Planck-Institute for Extraterrestrial Physics, Giessenbachstrasse 1, D-85748 Garching, Germany

${ }^{13}$ Department of Astronomy, University of Maryland, College Park, MD 20742, USA

14 Institute of Astronomy and Astrophysics, Academia Sinica, 645 N. Aohoku Place, Hilo, HI 96720, USA to determine the magnetic field morphology in the plane of the sky in the interstellar medium, especially in starforming regions. The morphology of magnetic fields has been studied via dust polarization over a wide range of scales: from Galactic scales (kpc; e.g., Stephens et al. 2011) all the way down to protostellar envelope scales (100s - 1000 s au; e.g., Girart et al. 2006; Rao et al. 2009; Stephens et al. 2013; Hull et al. 2014). Magnetic fields are thought to play a crucial role in the accretion process for protostellar disks via magnetorotational instability (e.g., Balbus \& Hawley 1998) and/or disk-winds (e.g., Blandford \& Payne 1982). Nevertheless, the magnetic field morphology remains observationally poorly constrained in the circumstellar environment.

The earliest attempts to detect polarization in circumstellar disks found polarization typically perpendicular to the major axis of the disk (Tamura et al. 1995, 1999). However, these observations were at $\sim 2000$ au resolution, so they did not resolve the disks; it is certainly possible that the polarized emission could come from protostellar envelopes. Hughes et al. (2009, 2013) made the first attempts to resolve submillimeter polarization within a disk, but such efforts resulted in non-detections, putting upper limits of the linear polarization fraction, $P=\sqrt{Q^{2}+U^{2}} / I$, at $\sim 1 \%$ when averaged over the telescope beam (where $I, Q$, and $U$ are Stokes parameters). Most recently, polarization has been detected toward a handful of disks and candidates disks: the Class 0 disk candidate IRAS 16293-2422 (Rao et al. 2014), the disk of Class I/II source HL Tau (Stephens et al. 2014; Kataoka et al. 2017), the Class 0 disk of L1527 (Segura-Cox et al. 2015), the Class 0 disk candidate of NGC 1333 IRAS 4A 
(Cox et al. 2015), the Herbig AE late-stage protoplanetary disk HD 142527 (Kataoka et al. 2016), and the disk candidate of the high-mass protostar Cepheus A HW2 (Fernández-López et al. 2016). Polarization toward disks have also been detected at mid-infrared wavelengths of 8.7, 10.3, and $12.5 \mu \mathrm{m}$ (Li et al. 2016, 2017). However, polarized emission at mid-infrared wavelengths can occur due to absorption, emission, and sometimes scattering, causing difficulty in interpreting the polarization morphology.

Despite these detections, the polarization morphologies usually were not consistent with what would be expected from magnetically aligned dust grains. In particular, Stephens et al. (2014) used the Combined Array for Research in Millimeter-wave Astronomy (CARMA) to measure the $1.25 \mathrm{~mm}$ polarization morphology in HL Tau. The morphology was inconsistent with grains aligned with the commonly expected toroidal magnetic fields (polarization/E-field vectors distributed radially in the disk). Instead, the E-vectors were oriented more or less along the minor axis of the disk. Kataoka et al. (2015, 2016) and Yang et al. (2016) suggested that the polarization morphology is actually consistent with that expected from self-scattering (also see Pohl et al. 2016; Yang et al. 2017). Indeed, several disks where polarization is detected show consistency with the polarization morphology expected from self-scattering rather than grains aligned with the magnetic field. However, except for the ALMA observations of HD 142527 (Kataoka et al. 2016) and HL Tau (Kataoka et al. 2017), the published observations are too coarse to resolve more than a few independent beams across the disk, making it difficult to distinguish between scattering and other polarization mechanisms.

The high-resolution ALMA observations of HD 142527 by Kataoka et al. (2016) resolved polarization for many 10s of independent resolution elements across the disk. The polarization was radial throughout most of the disk, which is expected for grains aligned with a toroidal field, but toward the edges the morphology changed from radial to azimuthal, which is more consistent with scattering. Models in Kataoka et al. (2016) found that scattering can broadly reproduce the features observed in parts of the disk - especially where the polarization orientations change sharply - but not everywhere. A complete understanding of this interesting case is still missing.

HL Tau is one of the brightest Class I/II disks in the sky at (sub)millimeter wavelengths, and thus the polarization morphology can be determined at high resolution with reasonable integration times. Kataoka et al. (2017) followed up on the Stephens et al. (2014) observations with $3.1 \mathrm{~mm}$ observations of HL Tau. Surprisingly, they found that the polarization morphology was azimuthal, which suggests grains aligned with their long axes perpendicular to the radiation field, as predicted by Tazaki et al. (2017, also see Lazarian \& Hoang 2007). Henceforth, we will call this grain alignment mechanism "alignment with the radiation anisotropy."

The very different polarization morphologies observed at $1.25 \mathrm{~mm}$ with CARMA (0.' 6 resolution, Stephens et al. 2014) and $3.1 \mathrm{~mm}$ with ALMA (0". 4 resolution, Kataoka et al. 2017) suggest that the morphology of the polarization emission is strongly dependent on the wavelength. The CARMA observations poorly constrained
$3.1 \mathrm{~mm}$ (ALMA Band 3)

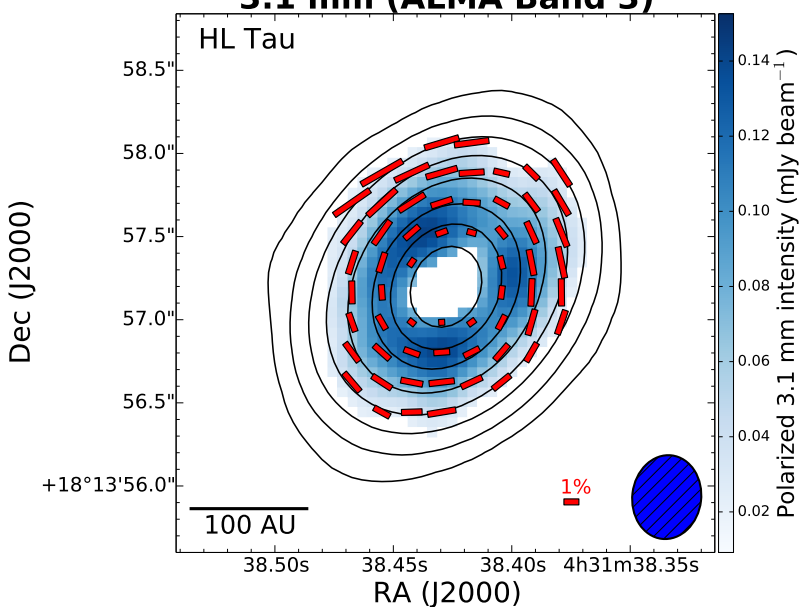

$1.3 \mathrm{~mm}$ (ALMA Band 6)

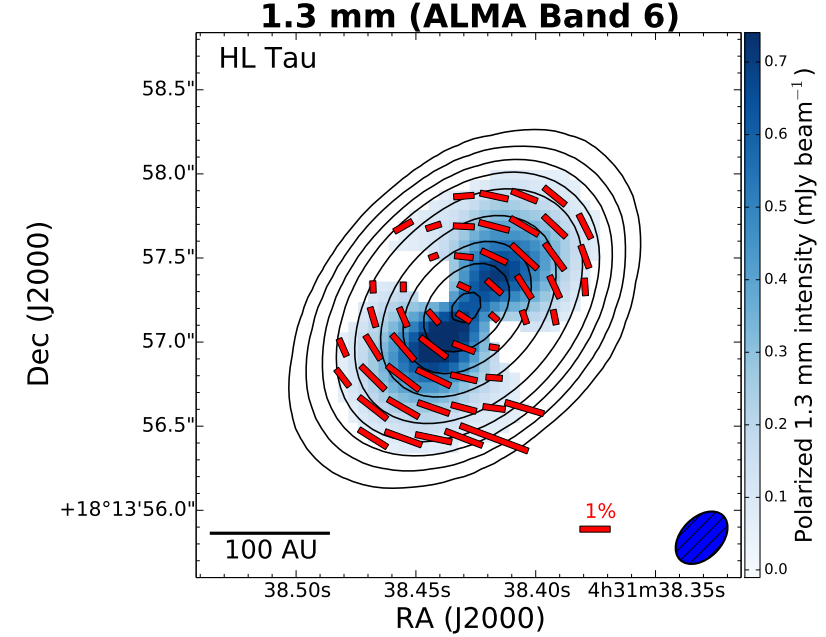

$870 \mu \mathrm{m}$ (ALMA Band 7)

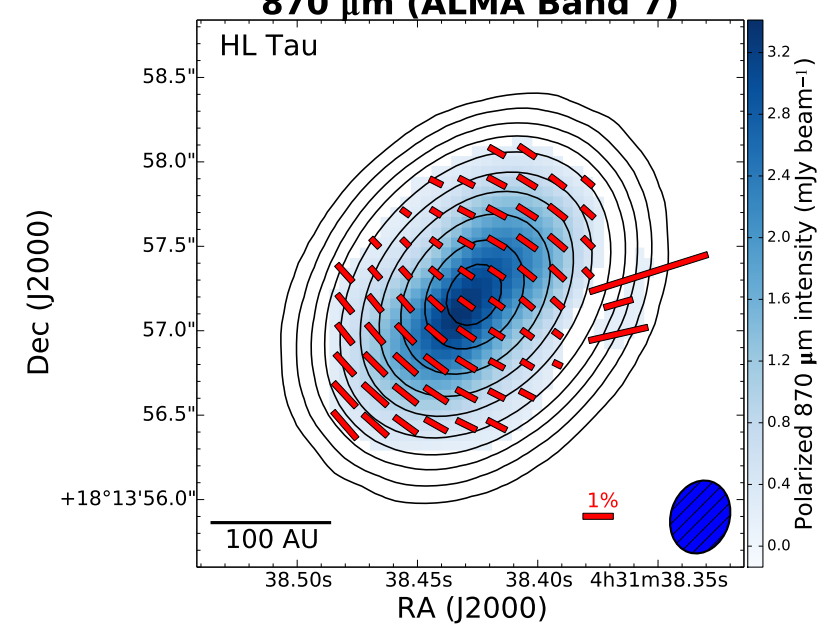

Figure 1. ALMA polarimetric observations at $3.1 \mathrm{~mm}$ (top, Kataoka et al. 2017), $1.3 \mathrm{~mm}$ (middle), and $870 \mu \mathrm{m}$ (bottom), where the red vectors show the $>3 \sigma$ polarization morphology (i.e., vectors have not been rotated). Vector lengths are linearly proportional to $P$. The color scale shows the polarized intensity, which is masked to only show $3 \sigma$ detections. Stokes $I$ contours in each panel are shown for $[3,10,25,50,100,200,325,500,750,1000] \times \sigma_{I}$, where $\sigma_{I}$ is 44,154 , and $460 \mu \mathrm{Jy} \mathrm{bm}^{-1}$ for $3.1 \mathrm{~mm}, 1.3 \mathrm{~mm}$, and $870 \mu \mathrm{m}$, respectively.

the $1.25 \mathrm{~mm}$ polarization morphology since they only re- 
solved polarization for $\sim 3-4$ independent beams across HL Tau. This paper presents ALMA observations at both $1.3 \mathrm{~mm}$ and $870 \mu \mathrm{m}$ at resolutions of $0 . .3$ and $0 .{ }^{\prime \prime} 4$, respectively.

\section{OBSERVATIONS}

With the ALMA Cycle 4 program 2016.1.00162.S (PI: I. Stephens), we observed polarized emission toward HL Tau at $870 \mu \mathrm{m}$ (Band 7) and at $1.3 \mathrm{~mm}$ (Band 6) on 2016 December 4 and 2017 July 12, respectively. The ALMA configurations for $870 \mu \mathrm{m}$ and $1.3 \mathrm{~mm}$ observations were C40-4 and C40-5, respectively. At $870 \mu \mathrm{m}$, the correlator was tuned to four $1.75 \mathrm{GHz}$ spectral windows at center sky frequencies of 336.5, 338.6, 348.5, and $350.5 \mathrm{GHz}$, and for $1.3 \mathrm{~mm}$ at center frequencies of 224 , 226,240 , and $242 \mathrm{GHz}$. For both wavelengths, the bandpass and flux calibrator was J0510+1800, the phase calibrator was J0431+1731, and the polarization calibrator was J0522-3627.

We used the delivered calibrated uv-data and performed imaging with CASA version 4.7.2. We used the CASA tclean task using a Briggs weighting parameter of robust $=1$. After a quick clean, we performed a series of phase-only self calibration, and images were cleaned after each self calibration iteration with progressively shorter solution intervals. We first performed several rounds of self calibration to the Stokes I image only, and then did self calibration a final round of self calibration on all four Stokes parameters. Images were primary-beam corrected. We also include $3.1 \mathrm{~mm}$ (Band 3 ) data which were from Kataoka et al. (2017), except we re-imaged in the identical way described above. The synthesized beams for $3.1 \mathrm{~mm}, 1.3 \mathrm{~mm}$, and $870 \mu \mathrm{m}$ were $0^{\prime \prime} .51 \times 0^{\prime \prime} 41$ with a position angle (measured counterclockwise from north) of $-67.6 ; 0^{\prime \prime} 37 \times 0^{\prime \prime} .24$ at $-44^{\circ} .1$; and $00^{\prime \prime} 44 \times 0^{\prime \prime} .35$ at -17.9 , respectively. Given a distance to HL Tau of $\sim 140 \mathrm{pc}$ (Rebull et al. 2004), the spatial resolution is 64,42 , and $55 \mathrm{au}$, respectively.

The ALMA instrumental error on $P$ is expected to be about $0.1 \%$. In cases where the measured $P$ rms was less than this value, we used $0.1 \%$ as our error. The polarization intensity and $P$ was de-biased via the method described in Hull et al. (2014) and Hull \& Plambeck (2015).

\section{RESULTS}

Figure 1 shows the observed polarization morphology for HL Tau for ALMA at $3.1 \mathrm{~mm}, 1.3 \mathrm{~mm}$, and $870 \mu \mathrm{m}$. The polarization morphology at $3.1 \mathrm{~mm}$ is azimuthal, with a void in the center about the size of the beam. This void likely occurs due to beam-averaging an azimuthal polarization morphology within the beam. The $3.1 \mathrm{~mm}$ polarization morphology is broadly consistent with the pattern expected for emission from grains aligned with radiation anisotropy. ${ }^{15}$ For $870 \mu \mathrm{m}$, the polarization morphology is extremely uniform and parallel with the minor axis of the disk. This morphology is predicted for Rayleigh scattering for a geometrically thin (dust) disk (Yang et al. 2017). Toward the west edge of the disk, there is polarization detected that is not aligned with the rest of these vectors. This emission is about the size of the beam and could possibly be a spurious $3 \sigma$ detection.

\footnotetext{
${ }^{15}$ We will postpone a detailed discussion of the caveats of this interpretation to a future publication.
}

The $1.3 \mathrm{~mm}$ morphology has slightly more structure but appears to be a superposition of those at $3.1 \mathrm{~mm}$ and $870 \mu \mathrm{m}$. Notably, along the disk's minor axis, the $3.1 \mathrm{~mm}$ vectors are perpendicular to the axis while the $870 \mu \mathrm{m}$ vectors are parallel. At $1.3 \mathrm{~mm}$, these cancel, and thus polarization is significantly undetected along the minor axis. On the contrary, along the disk's major axis, $3.1 \mathrm{~mm}$ and $870 \mu \mathrm{m}$ vectors are both perpendicular, and $P$ is highest on average for this line along the disk.

The transition of polarization morphologies from one wavelength to another is also apparent in the Stokes $Q$ and $U$ images, as seen in Figure 2. Specifically, at $3.1 \mathrm{~mm}$ the Stokes $Q$ image shows negative regions from north to south and positive regions from east to west. At $1.3 \mathrm{~mm}$, the negative regions are much brighter than the positive regions, and at $870 \mu \mathrm{m}$, the positive regions almost completely disappear. Stokes $U$ shows two positive and two negative regions at $3.1 \mathrm{~mm}$. The negative blobs disappear at $1.3 \mathrm{~mm}$, while the positive regions connect, and at $870 \mu \mathrm{m}$, the positive regions turn into a single, large region.

For the Stokes $V$ images (Figure 2), $3.1 \mathrm{~mm}$ appears to be purely noise. For both $1.3 \mathrm{~mm}$ and $870 \mu \mathrm{m}$, Stokes $V$ has a negative flux blob offset to the top-right of the Stokes $I$ intensity, and this blob is significantly brighter in $870 \mu \mathrm{m}$. Although circular polarization can in principle be produced by scattering of linearly polarized light off non-spherical grains (e.g., Tazaki et al. 2017), Stokes $V$ has not been well characterized for ALMA, so its detection could be spurious and will not be discussed in detail in this paper.

The central polarization vectors for $1.3 \mathrm{~mm}$ and $870 \mu \mathrm{m}$ are $55^{\circ} .4 \pm 0.96$ and $55^{\circ} .3 \pm 0.41$, respectively. The major axis of HL Tau's disk is $138^{\circ} .02 \pm 0.07$ (ALMA Partnership et al. 2015), indicating the polarization vectors are $\sim 7^{\circ}$ from being perpendicular to the major axis. We would expect the polarization to be perfectly perpendicular to the major axis. The reason for this small $7^{\circ}$ discrepancy is uncertain, but could indicate asymmetry in the disk.

The right panels of Figure 2 show $P$ for each wavelength. For $1.3 \mathrm{~mm}$ and $870 \mu \mathrm{m}$, there is a slight asymmetry for $P$ along the major axis of the disk, with the southeast more polarized than the northwest. For $1.3 \mathrm{~mm}$, the absolute difference between the two $P$ peaks along the major axis is $0.0013 \pm 0.0014$, which is not statistically significant. The asymmetry for $870 \mu \mathrm{m}$ is more apparent, although the southeast part of the disk does not peak (i.e., it is still increasing at the edge of the detected polarized emission). The $P$ peak toward the northwest is $0.0077 \pm 0.0010$. Drawing a line from the northwest peak through the center of the disk, at the same radius on the southeast side, $P=0.0094 \pm 0.0010$. $P$ rises to $\sim 0.0115$ at the edge of the disk. Therefore, the $P$ asymmetry along the major axis for $870 \mu \mathrm{m}$ is $\sim 0.002-0.004$. These asymmetries are relatively moderate, but the fact that they appear both at $1.3 \mathrm{~mm}$ and $870 \mu \mathrm{m}$ suggests they could be a real feature. If we consider total polarization $P_{T}=\sqrt{Q^{2}+U^{2}+V^{2}} / I$, the asymmetry is similar; for $1.3 \mathrm{~mm}$, it is $0.0012 \pm 0.0014$, while for $870 \mu \mathrm{m}$ it is $\sim 0.001-0.004$.

Yang et al. (2017) showed that asymmetry along the major axis is not expected for scattering-induced polar- 


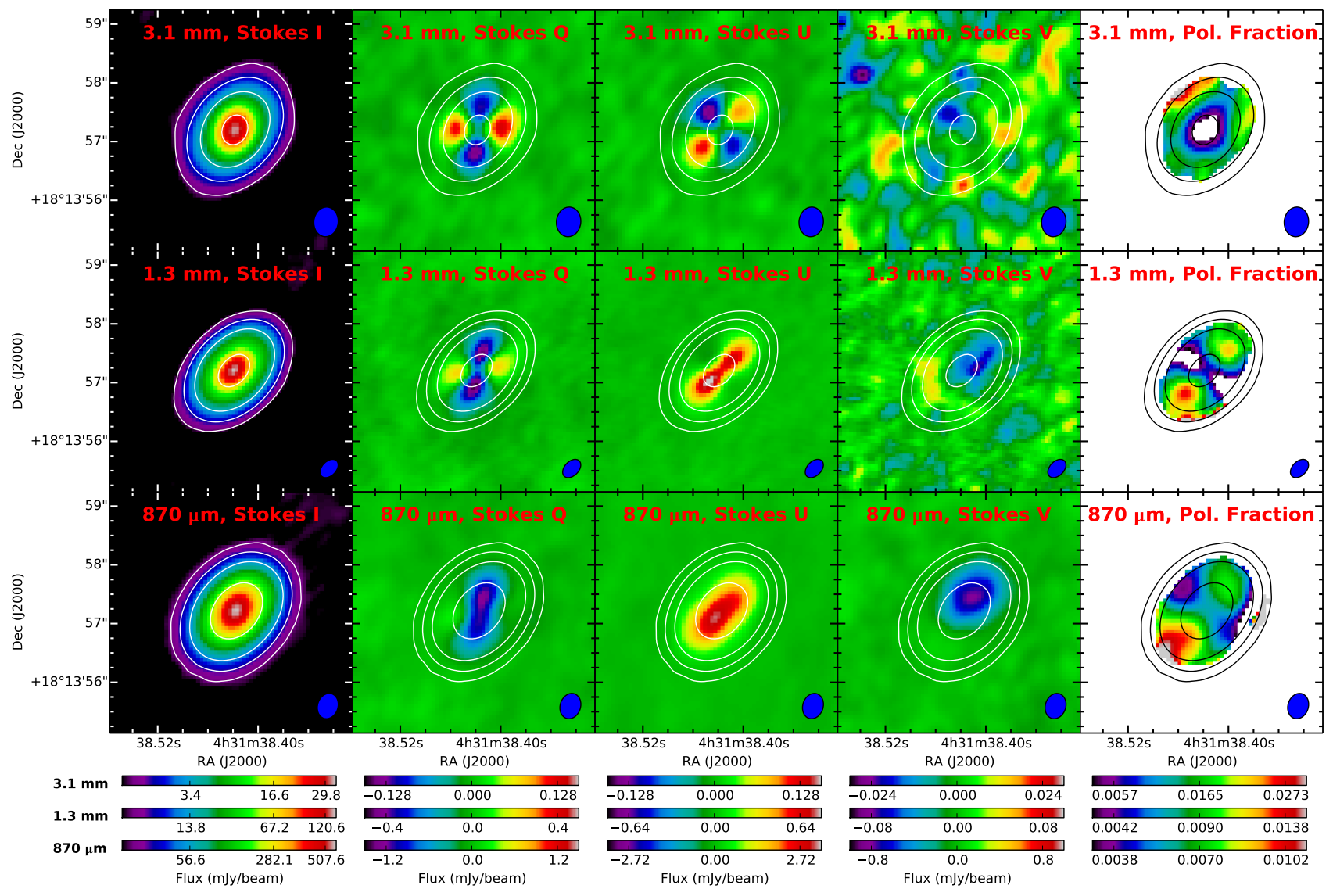

Figure 2. ALMA observations of the Stokes parameters $I Q U V$ and the linear polarization fraction $P$ for $3.1 \mathrm{~mm}$ (top), $1.3 \mathrm{~mm}$ (middle), and $870 \mu \mathrm{m}$ (bottom). Stokes $I$ contours for a particular wavelength is shown in each panel for $[4,25,100,500] \times \sigma_{I}$, where $\sigma_{I}$ is given in Figure 1.

ization in an axisymmetric disk, so axisymmetry must be broken if the polarization is really due to scattering even though the total intensity (Stokes $I$ ) is highly axisymmetric. On the other hand, the polarization along the minor axis is expected to be asymmetric if the dust disk is optically thick and has a significant angular width (for a cartoon illustration, see Figure 6 in Yang et al. 2017). HL Tau disk is known to be optically thick at $870 \mu \mathrm{m}$ (Carrasco-González et al. 2016), but no asymmetry is detected along the minor axis. The lack of asymmetry for $P$ along the optically thick minor axis implies that that the large grains responsible for scattering at $870 \mu \mathrm{m}$ are already settled into a thin layer (Yang et al. 2017). Evidence for dust settling in HL Tau was also found in Kwon et al. (2011, 2015) based on modeling of the millimeter dust continuum, and in Pinte et al. (2016) based on the shape of gaps in dust continuum images

\section{1.3 MM MORPHOLOGICAL MODEL OF HL TAU}

For illustrative purposes, we have also developed two simple models for the $1.3 \mathrm{~mm}$ data: one for the polarization orientation and the other for the spatial distribution of the polarized intensity.

The polarization orientation model is a simple 50-50 mix of a purely azimuthal pattern (mimicking the pattern observed at $3.1 \mathrm{~mm}$ ) and a uni-directional pattern along the minor axis (an idealization of the pattern observed at $870 \mu \mathrm{m})$. We created the model by averaging

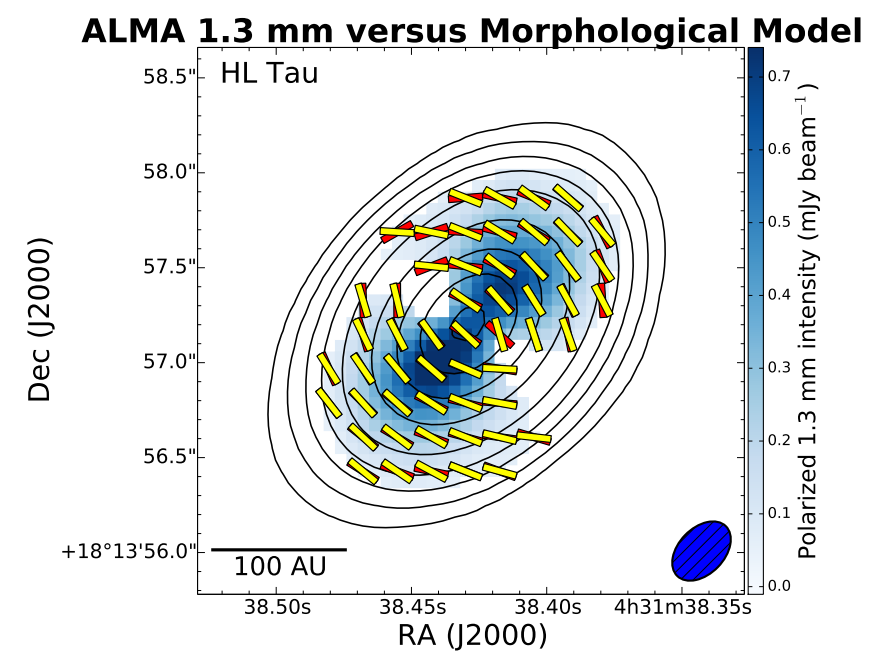

Figure 3. Morphological $1.3 \mathrm{~mm}$ model for a $50-50 \mathrm{mix}$ of azimuthal and uniform patterns is shown with yellow vectors, which are overlaid on top of the observed polarization morphology (red vectors). The contours are Stokes $I$, with the levels given in Figure 1.

the Stokes $Q$ and $U$ of the two patterns separately. Figure 3 shows the model compared with the observations. The two match remarkably well, which strengthens the notion that the $1.3 \mathrm{~mm}$ emission is a transition case between $3.1 \mathrm{~mm}$ and $870 \mu \mathrm{m}$. 
The polarized intensity model is based on a disk model similar to Model A of Yang et al. (2017), but with a reduced scale height for the dust layer. The grains are assumed to be oblate spheroids with their shortest axes aligned radially, as expected in the case of alignment with the radiation anisotropy. The intrinsic polarization fraction of the non-spherical grains is set to $1 \%$, and the effective grain size is $30 \mu \mathrm{m}$. The direct emission and scattering by such grains are computed under the electrostatic approximation (see Yang et al. 2016 for details). The resulting polarization pattern is shown in Figure 4 and broadly matches the observed pattern, especially the dumbbell-shaped distribution of the polarized intensity and the morphology of the polarization vectors. This model is not unique and does not match all details of the data; a detailed modeling will have many uncertainties in terms of dust properties and disk structure and is beyond the scope of this paper. Nevertheless, it does provide an illustration that the observed pattern at $1.3 \mathrm{~mm}$ can plausibly be explained with a combination of direct emission and scattering by aligned grains.

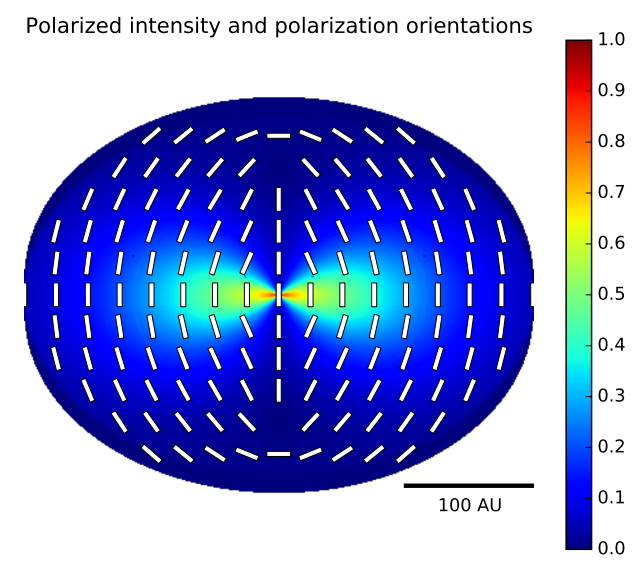

Figure 4. Distribution of polarized intensity with polarization vectors superposed for an illustrative disk model where the polarization is produced by a combination of direct emission and scattering by azimuthally aligned grains. Color scale has been normalized to have a peak of 1 .

\section{SUMMARY}

Kataoka et al. (2017) suggested that the polarization emission at $3.1 \mathrm{~mm}$ for HL Tau is caused by grain alignment via radiation anisotropy. In this paper, we present polarization images at two additional wavelengths. At $870 \mu \mathrm{m}$ the polarization morphology is uniform, which is consistent with polarization due to self-scattering. At $1.3 \mathrm{~mm}$, the polarization morphology appears to be a mix of the polarization morphologies at $3.1 \mathrm{~mm}$ and $870 \mu \mathrm{m}$, rather than purely self-scattering, as suggested in other studies (e.g., Kataoka et al. 2015, 2017; Yang et al. 2016). These observations are the first to show a clear transition in a disk's (sub)millimeter polarization morphology with wavelength. The observations also provide a clear confirmation of two different mechanisms causing polarization emission at (sub)millimeter wavelengths. These observations are not consistent with polarized emission from grains aligned with a toroidal magnetic field.

We are able to reproduce the morphology with a morphological model and a theoretical calculation. We also find tentative evidence that the linear polarization fraction $P$ is asymmetric along the major axis of the disk, which would suggest that HL Tau is not perfectly axisymmetric. Furthermore, the lack of asymmetry along the minor axis at the optically thick wavelength of $870 \mu \mathrm{m}$ may indicate that the large grains responsible for the scattering-induced polarization have already settled into a geometrically thin layer, which provides an independent check on the dust settling inferred from the shape of the gaps in HL Tau disk.

By observing high-resolution polarization of HL Tau at three different wavelengths, we have the ability to decipher which polarization mechanisms are occurring at different wavelengths. Without observing polarization of HL Tau at $3.1 \mathrm{~mm}$ and $870 \mu \mathrm{m}$, the polarization morphology at $1.3 \mathrm{~mm}$ would have been difficult to interpret. Understanding and precisely modeling polarization in a protostellar disk probably require multi-wavelength, high-resolution polarimetric data.

H.F.Y. is supported by an ALMA SOS award. Z.Y.L. is supported in part by NASA NNX14AB38G and NSF AST-1313083 and 1716259. A.K. acknowledges support by JSPS KAKENHI grant number JP15K17606. W.K. was supported by Basic Science Research Program through the National Research Foundation of Korea (NRF-2016R1C1B2013642). This paper makes use of the following ALMA data: ADS/JAO.ALMA\#2016.1.00115.S and ADS/JAO.ALMA\#2016.1.00162.S. ALMA is a partnership of ESO (representing its member states), NSF (USA) and NINS (Japan), together with NRC (Canada); MOST and ASIAA (Taiwan); and KASI (Republic of Korea), in cooperation with the Republic of Chile. The Joint ALMA Observatory is operated by ESO, AUI/NRAO and NAOJ. The National Radio Astronomy Observatory is a facility of the National Science Foundation operated under cooperative agreement by Associated Universities, Inc.

\section{REFERENCES}

ALMA Partnership, Brogan, C. L., Pérez, L. M., et al. 2015, ApJ, 808, L3

Andersson, B.-G., Lazarian, A., \& Vaillancourt, J. E. 2015, ARA\&A, 53, 501

Balbus, S. A., \& Hawley, J. F. 1998, Reviews of Modern Physics, 70,1

Blandford, R. D., \& Payne, D. G. 1982, MNRAS, 199, 883

Carrasco-González, C., Henning, T., Chandler, C. J., et al. 2016, ApJ, 821, L16

Cox, E. G., Harris, R. J., Looney, L. W., et al. 2015, ApJ, 814, L28

Fernández-López, M., Stephens, I. W., Girart, J. M., et al. 2016, ApJ, 832, 200

Girart, J. M., Rao, R., \& Marrone, D. P. 2006, Science, 313, 812

Hughes, A. M., Hull, C. L. H., Wilner, D. J., \& Plambeck, R. L. 2013, AJ, 145, 115

Hughes, A. M., Andrews, S. M., Espaillat, C., et al. 2009, ApJ, 698,131

Hull, C. L. H., \& Plambeck, R. L. 2015, Journal of Astronomical Instrumentation , 4, 1550005

Hull, C. L. H., Plambeck, R. L., Kwon, W., et al. 2014, ApJS,

213,13 
Kataoka, A., Muto, T., Momose, M., Tsukagoshi, T., \& Dullemond, C. P. 2016a, ApJ, 820, 54

Kataoka, A., Tsukagoshi, T., Pohl, A., et al. 2017, ApJ, 844, L5

Kataoka, A., Muto, T., Momose, M., et al. 2015, ApJ, 809, 78

Kataoka, A., Tsukagoshi, T., Momose, M., et al. 2016b, ApJ, 831, L12

Kwon, W., Looney, L. W., \& Mundy, L. G. 2011, ApJ, 741, 3

Kwon, W., Looney, L. W., Mundy, L. G., \& Welch, W. J. 2015, ApJ, 808, 102

Lazarian, A. 2007, J. Quant. Spec. Radiat. Transf., 106, 225

Lazarian, A., \& Hoang, T. 2007, MNRAS, 378, 910

Li, D., Pantin, E., Telesco, C. M., et al. 2016, ApJ, 832, 18

Li, D., Telesco, C. M., Zhang, H., et al. 2017, arXiv:1708.08026

Pinte, C., Dent, W. R. F., Ménard, F., et al. 2016, ApJ, 816, 25

Pohl, A., Kataoka, A., Pinilla, P., et al. 2016, A\&A, 593, A12

Rao, R., Girart, J. M., Lai, S.-P., \& Marrone, D. P. 2014, ApJ, 780, L6

Rao, R., Girart, J. M., Marrone, D. P., Lai, S.-P., \& Schnee, S. 2009, ApJ, 707, 921

Rebull, L. M., Wolff, S. C., \& Strom, S. E. 2004, AJ, 127, 1029
Segura-Cox, D. M., Looney, L. W., Stephens, I. W., et al. 2015, ApJ, 798, L2

Stephens, I. W., Looney, L. W., Dowell, C. D., Vaillancourt, J. E., \& Tassis, K. 2011, ApJ, 728, 99

Stephens, I. W., Looney, L. W., Kwon, W., et al. 2013, ApJ, 769, L15

Stephens, I. W., Looney, L. W., Kwon, W., et al. 2014, Nature, 514,597

Tazaki, R., Lazarian, A., \& Nomura, H. 2017, ApJ, 839, 56

Tamura, M., Hough, J. H., \& Hayashi, S. S. 1995, ApJ, 448, 346

Tamura, M., Hough, J. H., Greaves, J. S., et al. 1999, ApJ, 525, 832

Yang, H., Li, Z.-Y., Looney, L., \& Stephens, I. 2016a, MNRAS, 456, 2794

Yang, H., Li, Z.-Y., Looney, L. W., et al. 2016b, MNRAS, 460, 4109

Yang, H., Li, Z.-Y., Looney, L. W., Girart, J. M., \& Stephens, I. W. 2017, MNRAS, 472, 373 